$06,01,09$

\title{
Исследования физических свойств и электрокалорического эффекта в нано- и микрокерамике $\mathrm{BaTiO}_{3}$
}

\author{
(C) А.В. Карташев ${ }^{1,2}$, В.С. Бондарев ${ }^{1,3}$, И.Н. Флёров ${ }^{1,3}$, М.В. Горев ${ }^{1,3}$, Е.И. Погорельцев ${ }^{1,3}$, \\ A.В. Шабанов ${ }^{1}$, М.С. Молокеев ${ }^{1,3}$, S. Guillemet-Fritsch ${ }^{4}$, И.П. Раевский ${ }^{5}$ \\ ${ }^{1}$ Институт фризики им. Л.В. Киренского ФИЦ КНЦ СО РАН, \\ Красноярск, Россия \\ ${ }^{2}$ Красноярский государственный педагогический университет им. В.П. Астафьева, \\ Красноярск, Россия \\ ${ }^{3}$ Институт инженерной фризики и радиоэлектроники, Сибирский федеральный университет, \\ Красноярск, Россия \\ ${ }^{4}$ CIRIMAT, Universit de Toulouse \\ Toulouse, France \\ ${ }^{5}$ Научно-исследовательский институт физики, Южный федеральный университет, \\ Ростов-на-Дону, Россия \\ E-mail: akartashev@yandex.ru
}

Поступила в Редакцию 8 декабря 2018 г.

В окончательной редакции 8 декабря 2018 г.

Принята к публикации 15 января 2019 г.

Выполнены исследования теплоемкости, теплового расширения, диэлектрической проницаемости и электрокалорического эффекта (ЭКЭ) в объемных образцах $\mathrm{BaTiO}_{3}$ (ВТ) в виде нано $(\mathrm{nBT}-500 \mathrm{~nm})-$ и микро $(\mathrm{mBT}-1200 \mathrm{~nm})$-керамик, приготовленных соответственно плазменным методом Spark Plasma Sintering и твердофазным методом.

Размерный эффект в значительной мере проявился в подавлении аномалий теплоемкости и теплового расширения, в изменении температур и энтропий фазовых переходов, диэлектрической проницаемости и уменьшении максимальной величины интенсивного ЭКЭ: $\mathrm{nBT}-\Delta T_{\mathrm{AD}}^{\max }=29 \mathrm{mK}(E=2.0 \mathrm{kV} / \mathrm{cm})$, $\mathrm{mBT}-\Delta T_{\mathrm{AD}}^{\max }=70 \mathrm{mK}(E=2.5 \mathrm{kV} / \mathrm{cm})$. Рост проводимости при температурах выше $360 \mathrm{~K}$ приводит к значительному необратимому нагреву образцов за счет выделения джоулева тепла под электрическим полем, которое преобладает над ЭКЭ.

DOI: $10.21883 /$ FTT.2019.06.47689.309

\section{1. Введение}

Одной из важных задач физики конденсированного состояния является получение информации о влиянии полей разной физической природы на последовательности, энтропию и температуры фазовых переходов в твердых телах. В частности, это связано с поиском новых материалов, перспективных для применений в охлаждающих устройствах, работающих на основе калорических эффектов в твердых телах. Одно из перспективных направлений твердотельного охлаждения базируется на электрокалорическом эффекте (ЭКЭ), наиболее простом в плане технической реализации, что способствовало значительному росту числа публикаций, посвященных исследованию ЭКЭ [1-3].

ЭКЭ представляет собой изменение температуры или энтропии при приложении электрического поля соответственно в адиабатных или изотермических тепловых условиях. Как было показано [4], изменение температуры твердотельного хладагента за счет ЭКЭ на $2-5 \mathrm{~K}$ является достаточным для создания более энергоэффективной и коммерчески доступной системы охлаждения, чем существующие парожидкостные холодильные установки. Так как ЭКЭ пропорционален температурной производной поляризации $(d R / d T)_{E}$, то наибольшая его величина может быть реализована в сегнетоэлектрических материалах вблизи фазового перехода в сильных электрических полях $(E>200 \mathrm{kV} / \mathrm{cm})$. Однако электрический пробой в объемных сегнетоэлектриках наступает, как правило, уже в полях до $60 \mathrm{kV} / \mathrm{cm}$, что приводит к их разрушению. С другой стороны, тонкие сегнетоэлектрические пленки выдерживают более высокие электрические поля 1000-2000 kV/cm [5-7]. Именно по этой причине в пленках $\mathrm{PbZr}_{0.95} \mathrm{Ti}_{0.05} \mathrm{O}_{3}$ наблюдался гигантский интенсивный ЭКЭ $\Delta T_{\mathrm{AD}}^{\max }=12 \mathrm{~K}$ в электрическом поле $E=480 \mathrm{kV} / \mathrm{cm}$ [8]. Однако тонкие пленки имеют небольшую тепловую массу и не способны эффективно отводить электрокалорическую теплоту.

Другими перспективными сегнетоэлектрическими материалами с точки зрения реализации значительных величин ЭКЭ являются мультислойные структуры $[9,10]$. Они оказываются стойкими к электрическим полям высокой напряженности вследствие чередования в их объеме тонких слоев $(\sim 3 \mu \mathrm{m})$, а также обладают большой тепловой массой и, следовательно, высокой мощностью охлаждения. К сожалению, мультислойные структуры имеют низкий электрокалорический коэффициент $(\Delta T / \Delta E=1.7 \mathrm{mK} \cdot \mathrm{cm} / \mathrm{kV})$ по сравнению с 
объемными поликристаллическими сегнетоэлектриками $(\Delta T / \Delta E=30 \mathrm{mK} \cdot \mathrm{cm} / \mathrm{kV})$ из-за влияния размерных эффектов на параметры фазовых переходов и физические свойства $[11,12]$.

В связи с этим возникает закономерный вопрос, каким образом можно увеличить и электрокалорический коэффициент, и величину поля пробоя в сегнетоэлектрических материалах?

Как показано недавно [13-15], сильное влияние на устойчивость материала к электрическому пробою оказывает размер частиц микро- и нанокерамик. Исследования оксидных нанокристаллических керамических материалов, в частности керамики титаната бария $\mathrm{BaTiO}_{3}[16,17]$, продемонстрировали существенную зависимость параметров фазовых переходов и физических свойств от технологических условий синтеза и размера зерен [18]. Так, например, уменьшение толщины пленки от $1100 \mathrm{~nm}$ до $20 \mathrm{~nm}$ приводит к понижению температуры сегнетоэлектрического фазового превращения от $395 \mathrm{~K}$ до $371 \mathrm{~K}$. При этом аномалия теплоемкости размывается, а величина поляризации уменьшается от $11 \mu \mathrm{C} / \mathrm{cm}^{2}$ до $4 \mu \mathrm{C} / \mathrm{cm}^{2}$. В связи с этим становится принципиально важным вопрос о возможности управления размерами зерен в технологии получения керамических материалов. Исследования влияния размерных эффектов, термической предыстории материалов и методов их приготовления на электрофизические и термодинамические характеристики является актуальной задачей и с точки зрения нахождения оптимальных условий для повышения величины пробивного электрического поля и увеличения электрокалорической эффективности твердотельных хладагентов на основе микро и нанокерамических сегнетоэлектриков и мультислойных структур.

В настоящей работе представлены результаты исследований термодинамических (теплоемкости, теплового расширения), структурных, диэлектрических свойств и электрокалорического эффекта в микро- и нанокерамике $\mathrm{BaTiO}_{3}$ в широкой области температур. Такой комплексный подход к изучению тепловых и калорических свойств позволит получить важную информацию о природе физических явлений в микро- и нанокерамических материалах, о температурах фазовых переходов, энтропийных и деформационных параметров и калорической эффективности.

\section{2. Образцы и методы исследований}

Нанопорошки $\mathrm{BaTiO}_{3}$ были синтезированы путем осаждения оксалата. Процедура была подробно описана в работе [19]. В качестве прекурсора использовали $\mathrm{BaCl}_{2} \cdot 2 \mathrm{H}_{2} \mathrm{O}$ (Prolabo) и $\mathrm{TiOCl}_{2}$ (Prolabo). Прекурсор взвешивали в соответствующих пропорциях для контроля стехиометрии порошка, растворяли в воде и затем добавляли к этаноловому раствору щавелевой кислоты. Порошок $\mathrm{BaTiO}_{3}$ был получен после прокаливания при $850 \mathrm{C}$ в течение $4 \mathrm{~h}$ на воздухе. Порошки помещались в графитовый тигель с внутренним диаметром $8 \mathrm{~mm}$ и спекались плазменным методом (Spark Plasma Sintering (SPS)) с использованием системы SPS Sumimoto 2080 под давлением $75 \mathrm{MPa}$ в течение $3 \mathrm{~min}$ при $1150 \mathrm{C}$ (скорость нагрева $50 \mathrm{C} / \mathrm{min}$ ) с последующим быстрым охлаждением. Гранулы SPS отжигались в окислительной атмосфере в течение 48 часов при $850 \mathrm{C}$ для восстановления стехиометрии по кислороду. Основными преимуществами метода SPS по сравнению с обычным методом спекания являются более низкая температура и значительное уменьшение времени процесс, а также возможность получения весьма высокой плотности керамики $~ 97 \%$ при малых размерах зерен.

Микрокерамика $\mathrm{BaTiO}_{3}$ (mBT) была приготовлена по обычной твердотельной технологии. Стехиометрические количества $\mathrm{BaCO}_{3}$ и $\mathrm{TiO}_{2}$ (содержание основного вещества более 98\%) тщательно перемешивались в дистиллированной воде. После сушки полученной смеси твердофазный синтез проводили в течение двух четырехчасовых стадий при 1100 и $1150 \mathrm{C}$ соответственно. После каждой стадии продукт реакции измельчали и перемешивали. Для конечного спекания полученный порошок прессовали в виде дисков диаметром $30 \mathrm{~mm}$ и высотой $10 \mathrm{~mm}$ при $100 \mathrm{MPa}$. Трехпроцентный водный раствор поливинилового спирта использовали в качестве пластификатора. После выжигания пластификатора медленным нагревом до $600 \mathrm{C}$ спекание проводили при $1330 \mathrm{C}$ в течение 3 часов в закрытом глиноземном тигле на рифленой керамической подложке $\mathrm{ZrO}_{2}$. После спекания температуру снижали со скоростью $100 \mathrm{C} / \mathrm{h}$ до $800 \mathrm{C}$, а затем образцы медленно охлаждали в печи до комнатной температуры. Плотность керамики составляла $92.5 \%$ от теоретической.

Контроль состава и качества образцов проведен с помощью методов электронной микроскопии и рентгеноструктурного анализа.

Структурная морфология поверхности керамических образцов $\mathrm{BaTiO}_{3}$ исследовалась с помощью сканирующего электронного микроскопа (SEM) Hitachi TM3000 (Hitachi High-Technologies Co., Ltd., Tokyo, Japan). Типичное SEM-изображение нанокерамики $\mathrm{BaTiO}_{3}$ (nBT) представлено на рис. 1, $a$. На поверхности образца отчетливо видны отдельные гранулы с размерами 100-400 nm и небольшие каналы между ними. Повреждения гранул в виде трещин или вкраплений не наблюдались.

На снимке поверхности $\mathrm{mBT}$ (рис. 1,b) размерами примерно $1.5 \times 1.5 \mathrm{~mm}$, сложно различить отдельные частицы, т.е. нет выраженного рельефа, однако при большем увеличении (рис. 1,c), становятся отчетливо различимы гранулы с размерами 30-50 $\mu \mathrm{m}$.

Рентгеноструктурные исследования образцов nBT и $\mathrm{mBT}$ выполнены при комнатной температуре на дифрактометре D8-ADVANCE (излучение $\mathrm{CuK}_{\alpha}$, $\theta-2 \theta$-сканирование) с использованием линейного детекTopa VANTEC. Шаг сканирования по углу $2 \theta$ равен $0.016^{\circ}$, экспозиция - $0.3 \mathrm{~s}$ на шаг. Полученные рентгенограммы (рис. 2) использовались для уточнения структуры методом Ритвельда с использованием программы TOPAS 4.2 [20]. 

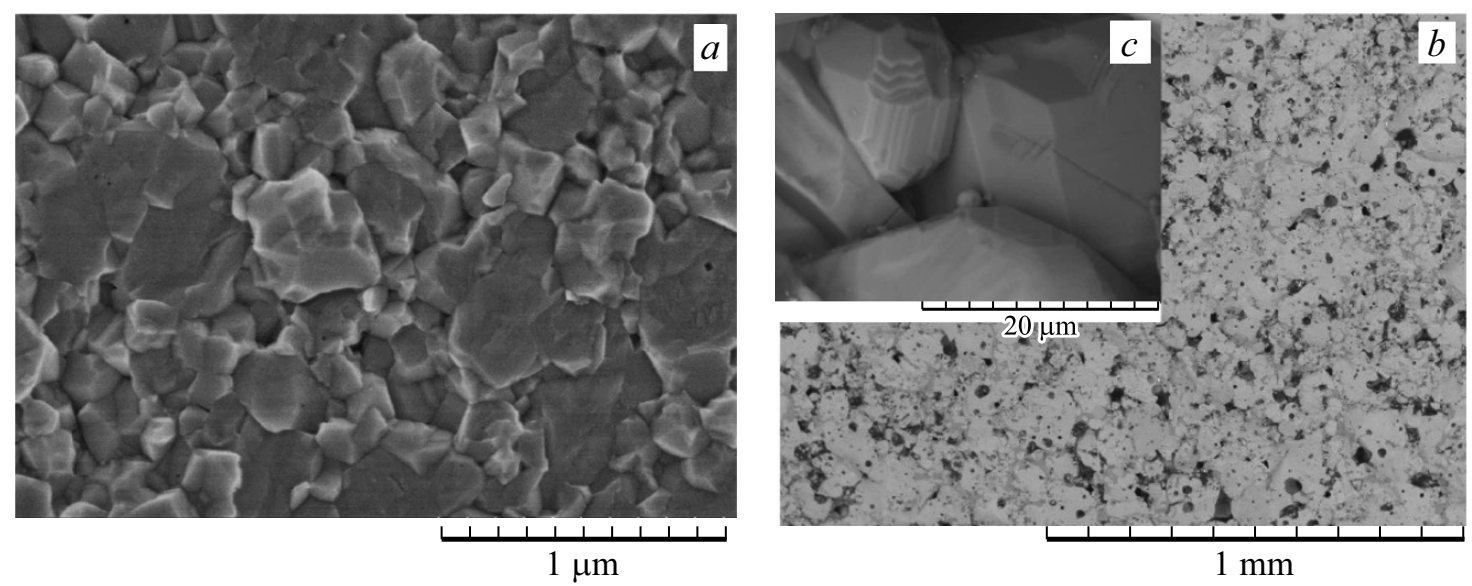

Рис. 1. SEM-изображение образцов $\mathrm{nBT}(a), \mathrm{mBT}(b, c)$.
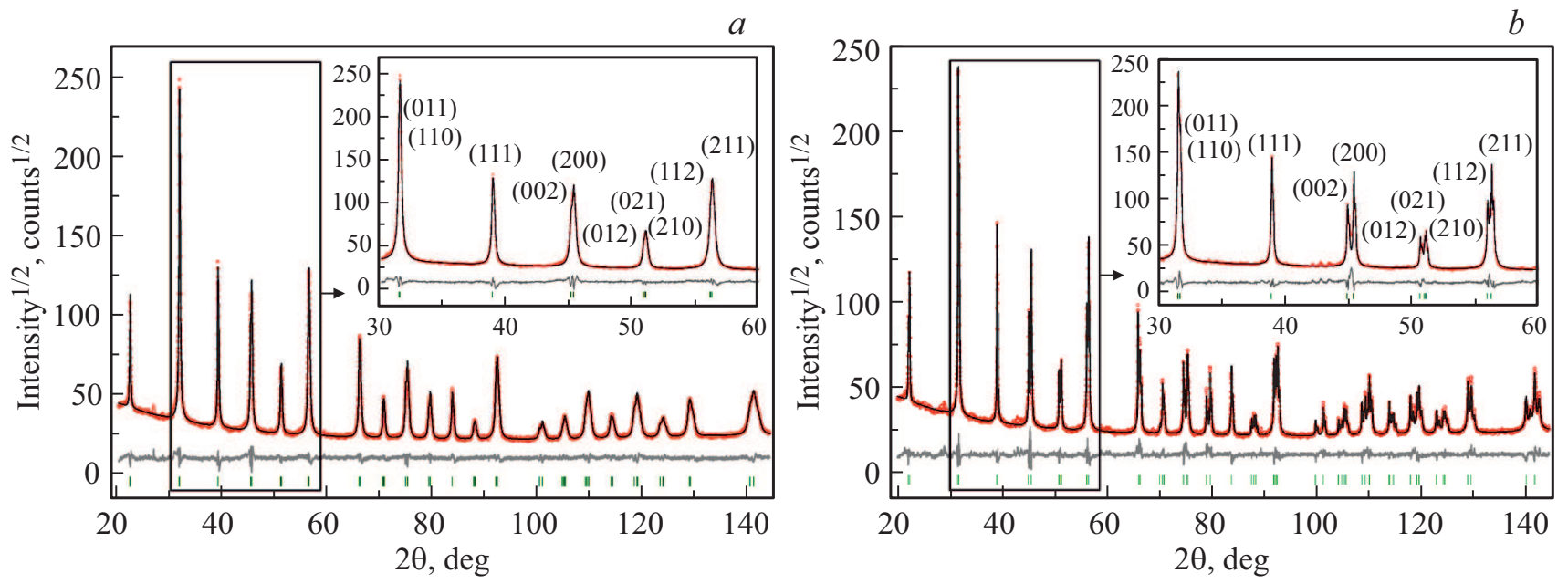

Рис. 2. Разностные рентгенограммы $\mathrm{nBT}(a)$ и $\mathrm{mBT}(b)$.

Все рефлексы надежно индексировались в тетрагональной ячейке $(P 4 m m)$ с параметрами, близкими к параметрам монокристалла $\mathrm{BaTiO}_{3}$ [21]. Эта кристаллическая структура была взята за исходную модель для уточнения методом Ритвельда. На дифрактограммах обоих образцов обнаружены лишь несколько небольших пиков, которые могут соответствовать примесным фазам с массой менее $1-2 \%$. Узкие и широкие основные рефлексы, наблюдавшиеся соответственно для микро- и нанокерамики, согласуются с ожидаемым размером кристаллитов. Оцененные значения усредненного размера кристаллитов действительно демонстрируют различные значения (табл. 1).

Окончательные уточнения всех параметров оказались стабильными с низкими значениями $R$-факторов (табл. 1 , рис. $2, a, b)$. Следует отметить, что параметры ячеек микро- и нанокерамических образцов $\mathrm{BaTiO}_{3}$ различны, что привело к увеличению объема элементарной ячейки в nBT (табл. 1). Анализ длин связей показал, что расстояния $d(\mathrm{Ba}-\mathrm{O})$ очень близки для обоих соединений в отличие от расстояний $d(\mathrm{Ti}-\mathrm{O})$. Можно предположить, что состав нано- и микрокристаллических соединений отличается, и это различие может быть связано с образованием вакансий.

Таблица 1. Основные результаты обработки рентгеноструктурных исследований нано- и микро- $\mathrm{BaTiO}_{3}$

\begin{tabular}{c|c|c}
\hline Соединение & $\mathrm{nBT}$ & $\mathrm{mBT}$ \\
\hline Sp.Gr. & $P 4 m m$ & $P 4 m m$ \\
$a, \AA$ & $4.00209(6)$ & $3.99326(2)$ \\
$c, \AA$ & $4.01915(8)$ & $4.03517(3)$ \\
$V, \AA^{3}$ & $64.374(2)$ & $64.3454(8)$ \\
$Z$ & 1 & 1 \\
$2 \theta$-interval, & $20-144$ & $20-144$ \\
$R_{w p}, \%$ & 6.15 & 8.08 \\
$R_{p}, \%$ & 4.73 & 5.90 \\
$R_{\text {exp }} \%$ & 2.87 & 2.98 \\
$\chi^{2}$ & 2.15 & 2.71 \\
$R_{\mathrm{B}}, \%$ & 1.39 & 2.93
\end{tabular}



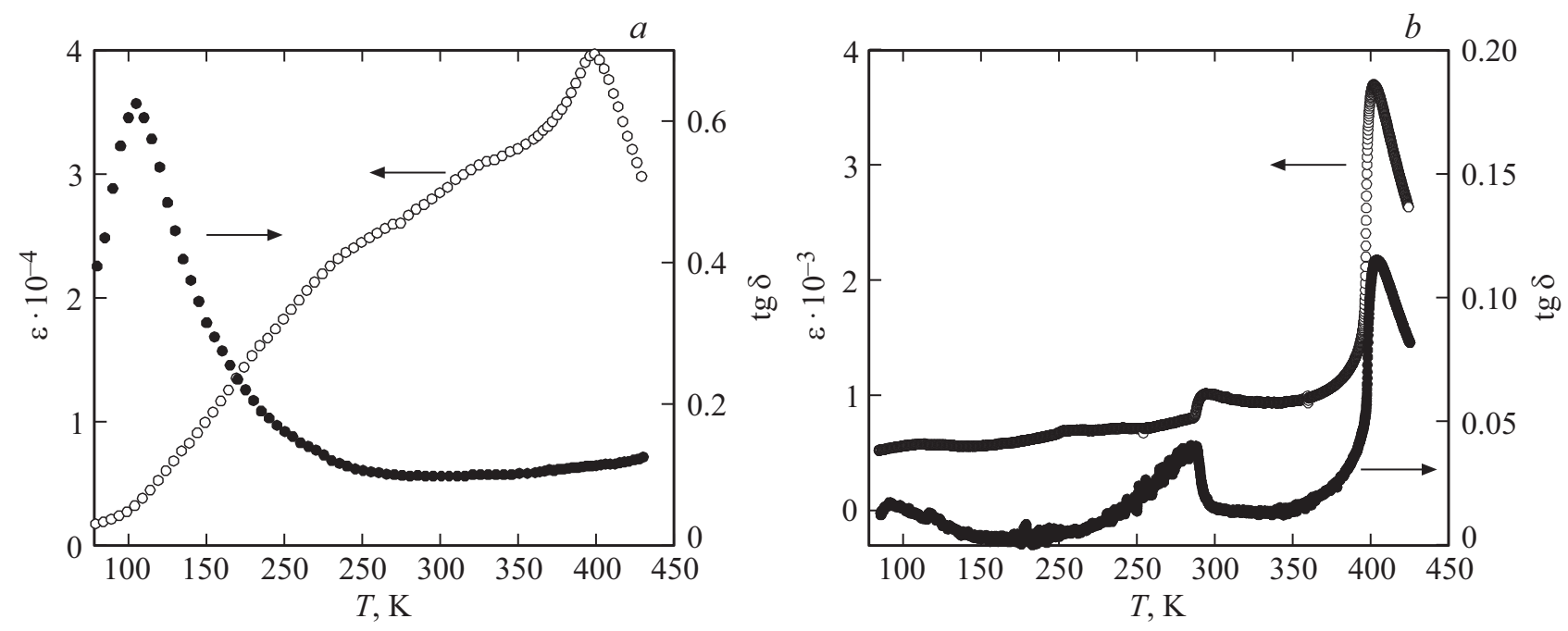

Рис. 3. Температурные зависимости диэлектрической проницаемости и тангенса потерь для нано- $(a)$ и микро- $(b)$ керамики $\mathrm{BaTiO}_{3}$.

Измерения диэлектрических свойств были выполнены с помощью прибора Е7-20 на частоте $1 \mathrm{kHz}$ для нанокерамики и $5 \mathrm{kHz}$ для микрокерамики. В качестве электродов для $\mathrm{nBT}$ использовалось золото, а для $\mathrm{mBT}-$ токопроводящая паста на основе серебра. Измерения были выполнены в режиме нагрева со скоростью в пределах $(0.5-2) \mathrm{K} / \mathrm{min}$ в температурном интервале $80-420 \mathrm{~K}$.

Дилатометрические исследования проводились на керамических образцах с размерами $L \approx 5 \mathrm{~mm}$ на индукционном дилатометре DIL-402C (Netzsch) в потоке сухого газообразного гелия. Скорости нагрева образца в интервале температур 100-700 К варьировались от 2 до $4 \mathrm{~K} / \mathrm{min}$. Эталон из плавленого кварца был использован для калибровки и учета расширения измерительной системы. Данные, полученные в нескольких сериях измерений, согласовывались между собой в пределах $2-3 \%$.

Исследования теплоемкости в температурном диапазоне 90-430 К выполнены на автоматизированном адиабатическом калориметре, подробно описанном в работе [22]. Скорости непрерывных нагревов варьировались в пределах от 0.01 до $1 \mathrm{~K} / \mathrm{min}$, время дискретных нагревов составляли от 20 до $40 \mathrm{~min}$. Образцы помещались в ячейку и фиксировались с помощью смазок ApiezonN и ApiezonH, которые обеспечивали надежный тепловой контакт. Для получения калориметрических данных в области высоких температур, $T>370 \mathrm{~K}$ измерения были выполнены с помощью прибора DSC 204 (Netzsch).

\section{3. Экспериментальные результаты и обсуждение}

\section{1. Диэлектрическая проницаемость}

Температурные зависимости диэлектрической проницаемости и тангенса угла потерь для образцов $\mathrm{nBT}$ и $\mathrm{mBT}$ представлены на рис. 3.
В случае нанокерамики (рис. $3, a$ ) наблюдается монотонный рост диэлектрической проницаемости, максимум пика которой при температуре $T_{1}=399 \mathrm{~K}$ достигает гигантских значений $\varepsilon=39600$. В области трех фазовых переходов (ФП) $\mathrm{BaTiO}_{3}$ наблюдаются сильно размытые аномалии $\varepsilon(T)$. При этом тангенс угла потерь в области низких температур имеет максимум с величиной 0.63 при $T=105 \mathrm{~K}$, затем монотонно уменьшается, достигая минимума $\sim 0.1$ в области температур $290-300 \mathrm{~K}$, и снова начинает плавно возрастать до величины 0.12 при максимальной температуре эксперимента. Важно отметить, что в области температур, соответствующих трем ФП, на графике диэлектрических потерь нет каких либо особенностей. Такое поведение $\operatorname{tg}(T)$ наблюдалось в нанокерамике $\mathrm{BaTiO}_{3}$ и ранее $[23,24]$ и соответствует температурной активации диэлектрической релаксации. Подобные явления были ранее обнаружены в хорошо известном материале ССТО, обладающем колоссальной диэлектрической проницаемостью [25].

Совсем другое поведение диэлектрических свойств наблюдается для микрокерамики (рис. $3, b$ ). На зависимости $\varepsilon(T)$ обнаружены три аномалии, соответствующие $\Phi П$ в $\mathrm{BaTiO}_{3}$. При температуре перехода между кубической и тетрагональной фазами $T_{1}=402 \mathrm{~K}$ максимум диэлектрической проницаемости достигает $\varepsilon=3700$. Диэлектрические потери в области ФП при $T_{2}=287 \mathrm{~K}$ и $T_{1}=403 \mathrm{~K}$ имеют ярко выраженные особенности. В районе $100 \mathrm{~K}$, также как и в случае $\mathrm{nBT}$, имеется максимум на зависимости $\operatorname{tg} \delta(T)$. Колоссальное различие в величинах $\varepsilon$ и поведении $\varepsilon(T)$ для нано- и микрокерамики может быть связано с различием в методике получения этих образцов. SPS-процесс эффективен при удалении резистивной компоненты в границах зерен, которая обычно наблюдается в традиционно спеченной керамике $\mathrm{BaTiO}_{3}$, что приводит к значительному росту диэлектрической проницаемости в нанокерамике изготовленной 
Таблица 2. Величины термодинамических параметров для $\mathrm{nBT}$ и $\mathrm{mBT}$ полученные на адиабатическом калориметре и DSC-204

\begin{tabular}{|c|c|c|c|c|c|c|c|}
\hline & & \multicolumn{2}{|c|}{ Куб. ↔ тетраг. } & \multicolumn{2}{|c|}{ Тетраг. $\leftrightarrow$ ромбич. } & \multicolumn{2}{|c|}{ Ромбич. ↔ орторомб. } \\
\hline & & $\mathrm{nBT}$ & $\mathrm{mBT}$ & $\mathrm{nBT}$ & $\mathrm{mBT}$ & $\mathrm{nBT}$ & $\mathrm{mBT}$ \\
\hline \multirow{2}{*}{ Adiabat } & $T, \mathrm{~K}$ & $383 \pm 2$ & $396.7 \pm 0.5$ & $310 \pm 2$ & $286.0 \pm 0.5$ & $227 \pm 2$ & $201.6 \pm 0.1$ \\
\hline & $\Delta S(\mathrm{~J} / \mathrm{molK})$ & 0.6 & 1.3 & 0.25 & 0.65 & 0.1 & 0.23 \\
\hline \multirow{2}{*}{ DSC } & $T, \mathrm{~K}$ & $380 \pm 5$ & $400.1 \pm 0.5$ & $311.0 \pm 2$ & $285.6 \pm 0.5$ & $222 \pm 2$ & $203.3 \pm 0.5$ \\
\hline & $\Delta S(\mathrm{~J} / \mathrm{molK})$ & - & 0.6 & - & 0.3 & $\begin{array}{c}1.2 \\
\text { (суммарная) }\end{array}$ & 0.2 \\
\hline
\end{tabular}

SPS-методикой [26]. Другими словами, нанокерамика высокой плотности (> 98\% от теоретической) содержит в себе меньшее количество резистивной компоненты по сравнению с микрокерамикой и тем самым имеет меньшую плотность свободных зарядов на границах зерен. В такой нанокерамике уменьшение размера зерна не меняет тип носителей заряда, связанных в основном с кислородными вакансиями, но при повторном окислении приводит к более значительному уменьшению плотности таких носителей, что сказывается в значительной мере на транспортном механизме проводимости, регулируемом, в основном, границами зерен [27].

\section{2. Тепловое расширение}

Результаты измерений теплового расширения микрои нанокерамических образцов $\mathrm{BaTiO}_{3}$ представлены на рис. 4. Аномалии $\alpha(T)$ в $\mathrm{mBT}$, связанные с фазовыми переходами $P m 3 m-P 4 m m-C 2 m m-R 3 m$, были найдены при температурах $T_{1}=400 \pm 2 \mathrm{~K}, T_{2}=290 \pm 2 \mathrm{~K}$, $T_{3}=206 \pm 2 \mathrm{~K}$ и достаточно близки к температурам переходов в кристалле $\mathrm{BaTiO}_{3}$, а величина коэффициента теплового расширения согласуется с данными [28,29].

Как видно из рис. 4 , в пBТ все аномалии $\alpha(T)$ сильно размыты, особенно аномалия в области низкотемпера-

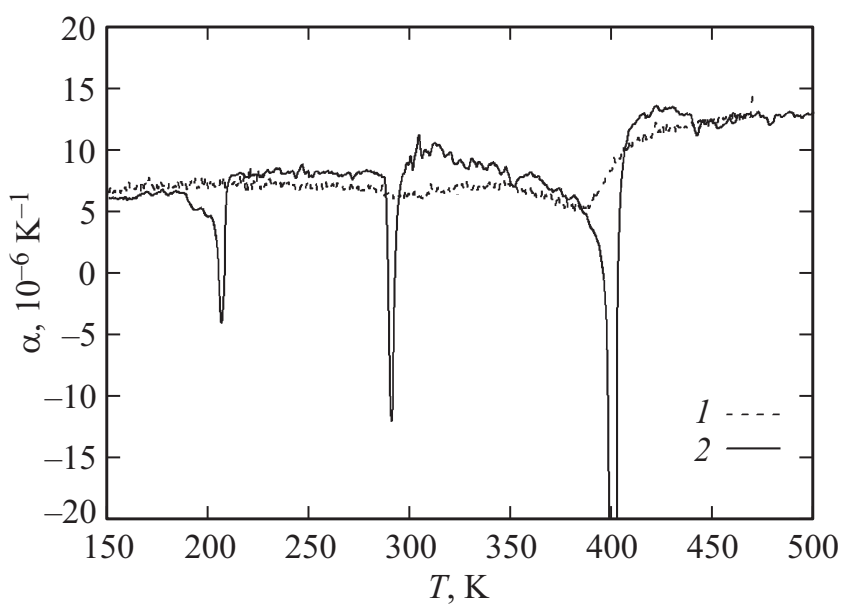

Рис. 4. Температурные зависимости коэффициента теплового расширения $\mathrm{nBT}(1)$ и $\mathrm{mBT}(2)$ керамики. турного фазового перехода $C 2 m m-R 3 m$. Уменьшение размера частиц (зерна) приводит также к понижению температуры Кюри для фазового перехода $P m 3 m-P 4 m m$ $T_{1}=384 \pm 5 \mathrm{~K}$. Изменение температур фазовых переходов $P 4 m m-C 2 m m$ и $C 2 m m-R 3 m$ не столь очевидны $T_{2}=303 \pm 5 \mathrm{~K}, T_{3}=205 \pm 15 \mathrm{~K}$.

\section{3. Теплоемкость нано- и микрокерамики}

Температурные зависимости теплоемкости $\mathrm{nBT}$ и mBT в интервале $80-430 \mathrm{~K}$ представлены на рис. 5, $a$ и $6, a$. В случае $\mathrm{nBT}$ на зависимости $C_{p}(T)$ имеется несколько небольших аномалий с размытыми пиками. Напротив, результаты на $\mathrm{mBT}$ демонстрируют три отчетливо различимые аномалии. Для выделения аномальной составляющей теплоемкости и вычисления энтропии фазовых переходов решеточная теплоемкость была определена аппроксимацией экспериментальных данных вне области существования аномалий с использованием комбинации функций Дебая и Эйнштейна. Вычисленные из этих данных избыточные теплоемкости $\Delta C_{p}$ (разность полной и решеточной) представлены на рис. $5, b$ и $6, b$ соответственно для $\mathrm{nBT}$ и $\mathrm{mBT}$. Температурные зависимости аномальной энтропии, связанные с последовательными ФП, были рассчитаны путем интегрирования функции $\left(\Delta C_{p} / T\right)(T)$ (рис. $5, c$ и рис. $\left.6, c\right)$.

Сравнение результатов, полученных для $\mathrm{nBT}$ и $\mathrm{mBT}$ показывает, что уменьшение размера зерна приводит к существенному размытию аномалий, связанных с ФП, к сужению температурной области существования тетрагональной фазы и уменьшению энтропий ФП (табл. 2) Эти результаты согласуются с данными исследований размерных эффектов в тонких пленках [30]. Однако в работе [31] при исследовании на установке DSC 204 (Netzsch) теплоемкости наноструктурированной керамики $\mathrm{BaTiO}_{3}$ с размерами зерен 50-1200 nm, полученной силовым воздействием в сочетании со сдвиговой деформацией, наблюдалось существенное увеличение энтропии (в несколько раз!) фазовых превращений в образце с наночастицами по сравнению с образцом, состоящем из микрочастиц. Такое увеличение энтропии (и соответственно поляризации) авторы связывали с большим количеством дефектов, содержащихся в кристаллитах. 


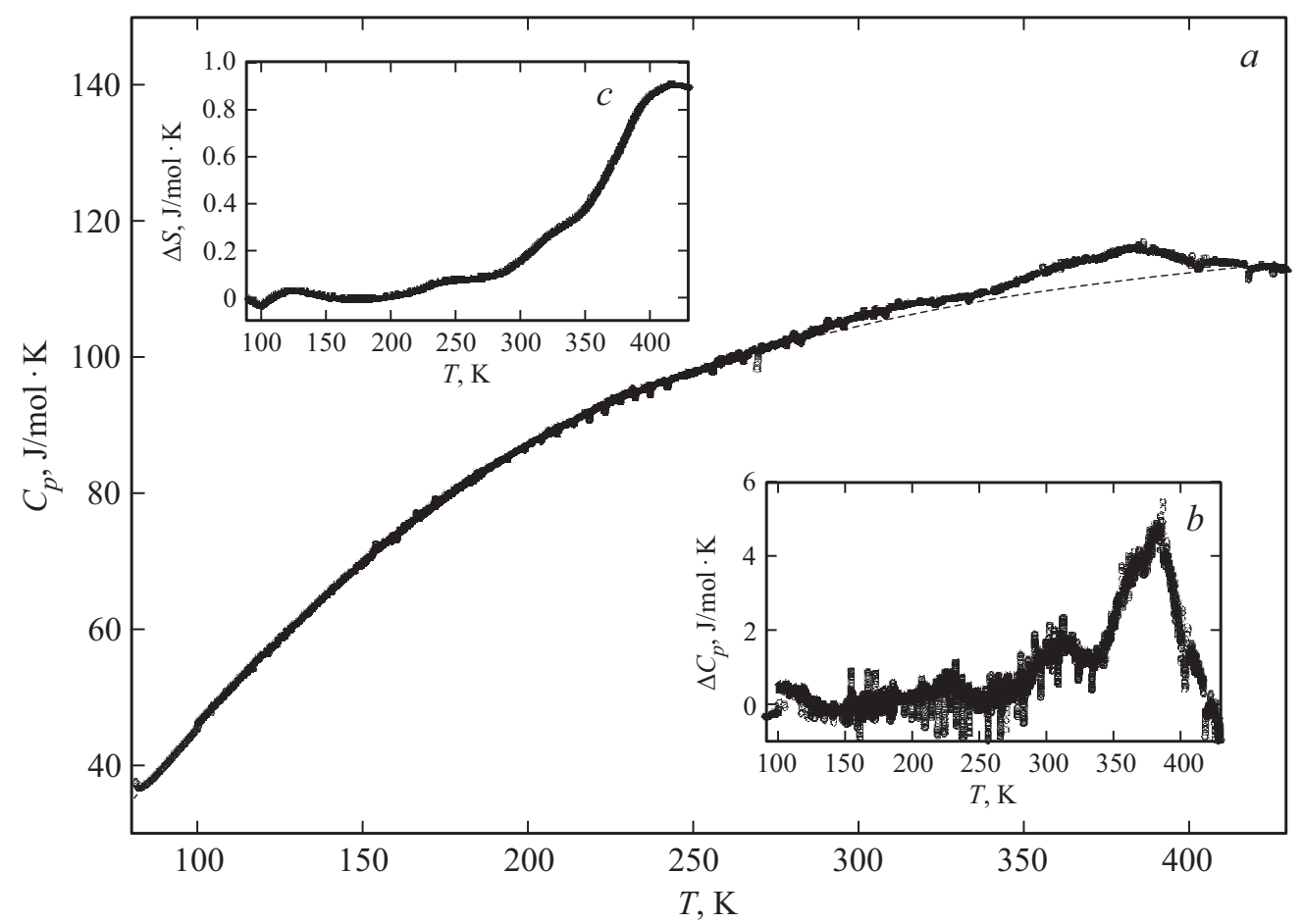

Рис. 5. Температурные зависимости теплоемкости $(a)$, избыточной теплоемкости $(b)$ и энтропии $(c) \mathrm{nBT}$.

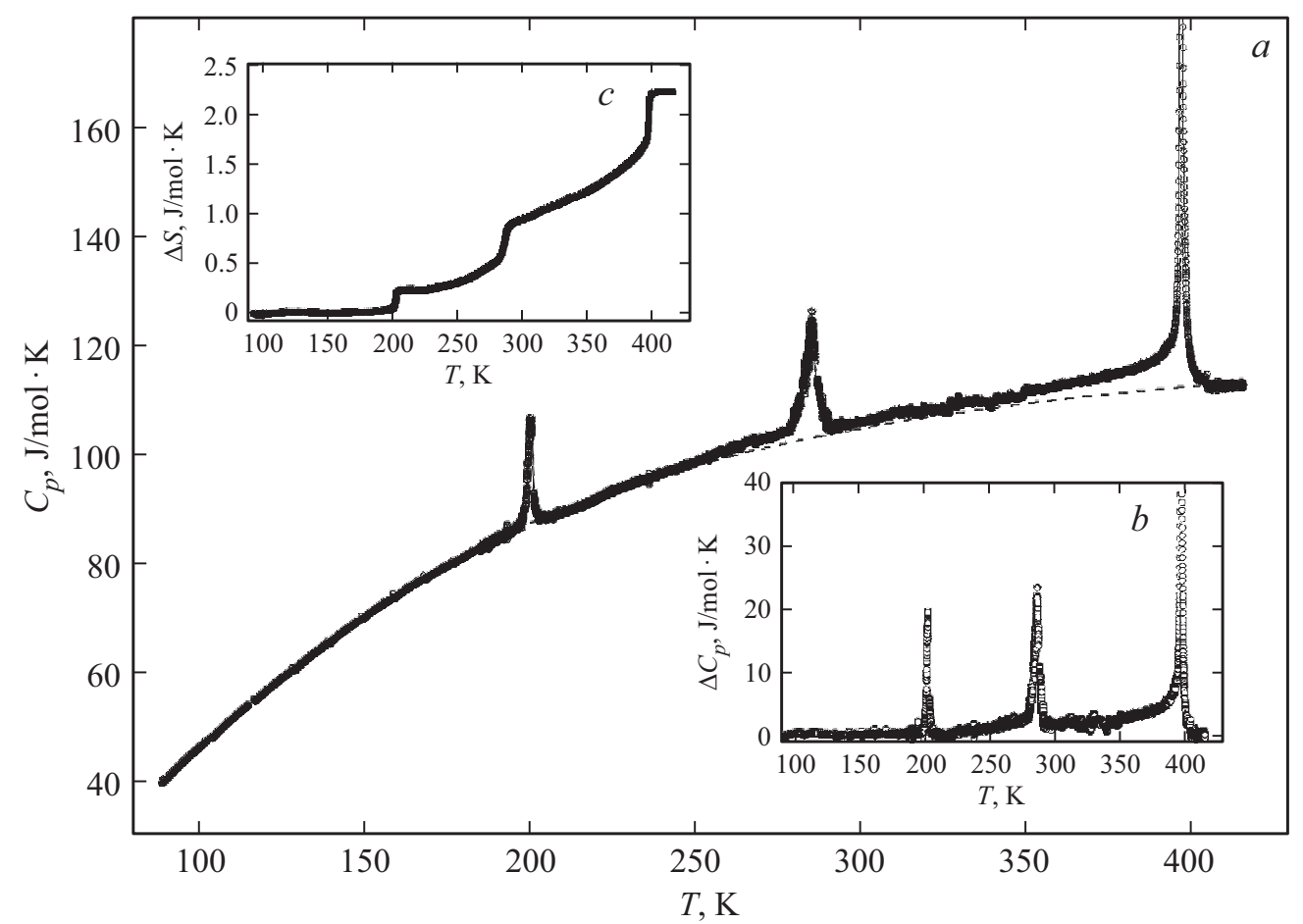

Рис. 6. Температурные зависимости теплоемкости $(a)$, избыточной теплоемкости $(b)$ и энтропии $(c) \mathrm{mBT}$.

При повторных измерениях наблюдалось уменьшение величины аномальной теплоемкости (а значит и энтропии), обусловленное температурным отжигом дефектов. Такое явное противоречие результатов вынудило нас провести дополнительные исследования на аналогич- ном дифференциальном сканирующем калориметре. На рис. 7 представлены температурные зависимости теплоемкости для $\mathrm{nBT}$ и $\mathrm{mBT}$.

В табл. 2 сведены значения термодинамических параметров, полученные на адиабатическом и дифферен- 
Таблица 3. Величины измеренного и реального электрокалорического эффекта и скорости паразитного разогрева в моменты приложения поля

\begin{tabular}{c|c|c|c|c|c|c|c}
\hline \multicolumn{2}{c|}{ Температура, $\mathrm{K}$} & 318.584 & 337.39 & 340.40 & 380.1 & 411.85 & 419.5 \\
\hline \multirow{2}{*}{$\Delta T_{\text {exр }}(\mathrm{mK})$} & $\mathrm{nBT}$ & 2.5 & - & 3.5 & - & 16 & - \\
\cline { 2 - 8 } & $\mathrm{mBT}$ & - & 4.5 & - & 14 & - & 10 \\
\hline \multirow{2}{*}{$\Delta T_{\mathrm{AD}}(\mathrm{mK})$} & $\mathrm{nBT}$ & 4.2 & - & 5.9 & - & 29 & - \\
\cline { 2 - 8 } & $\mathrm{mBT}$ & - & 15 & - & 46 & - & 33 \\
\hline $\begin{array}{l}\text { Скорость } \\
\text { нагрева, } \mathrm{mK} / \mathrm{min}\end{array}$ & $\mathrm{nBT}$ & $<0.05$ & - & 0.6 & - & 30 & - \\
\cline { 2 - 9 } & $\mathrm{mBT}$ & - & 5.0 & - & 65 & - & 410
\end{tabular}

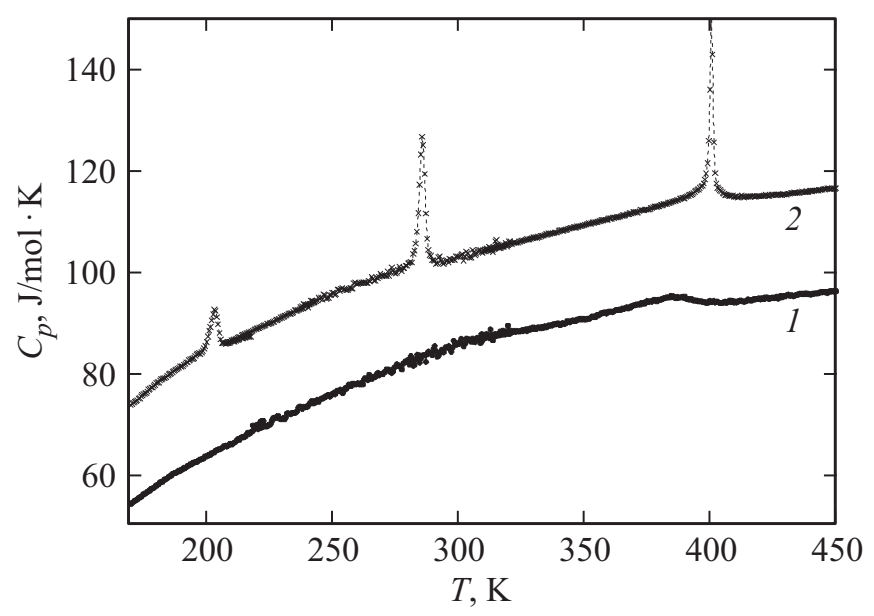

Рис. 7. Температурные зависимости теплоемкости для nBT (1) и $\mathrm{mBT}$ (2) полученные на DSC 204 (Netzsch).

циальном сканирующем калориметрах. В целом можно считать согласие результатов вполне удовлетворительным. Некоторые отличия в величинах температур и энтропий ФП связаны с различной точностью и условиями измерений на двух установках.

\section{4. Электрокалорический эффект}

Размерный эффект в значительной мере проявился в подавлении аномалий теплоемкости и теплового расширения, в изменении температур и энтропий фазовых переходов, диэлектрической проницаемости.

Полученные экспериментальные данные о тепловых и диэлектрических свойствах нано- и микрокерамики указывают на ярко выраженный размерный эффект, проявляющийся в понижении и размытии температуры ФП. В соответствии с данными [32,33] большая величина диэлектрической проницаемости в нанокерамике по сравнению с микрокерамикой, должна способствовать реализации значительного электрокалорического эффекта. Однако суммарная избыточная энтропия фазовых переходов в nBT, во-первых, меньше, чем в $\mathrm{mBT}$, и, во-вторых, она размыта в широком интервале температур, что может привести к уменьшению величины ЭКЭ.

Прямые измерения интенсивного ЭКЭ $\Delta T_{\mathrm{AD}}(T)$ выполнены на адиабатическом калориметре по методике, использованной нами ранее при исследовании электро- и магнетокалорического эффектов $[22,34,35]$. Для создания электрического поля использовался источник стабилизированного высокого напряжения. Теплоемкость электродов и подводящих проводов составляла менее $1 \%$ от суммарной теплоемкости (образец+ячейка), поэтому потери ЭКЭ на их нагрев не учитывались.

При определенной температуре адиабатический калориметр настраивался так, чтобы дрейф температуры образца был не хуже $\pm 10^{-3} \mathrm{~K} / \mathrm{min}$. Такое поведение температуры означает, что в криостате реализованы условия, максимально близкие к адиабатным: система стабилизирована в устойчивом динамическом равновесии. Затем к электродам образца прикладывался импульс электрического напряжения продолжительностью 3-10 min, что приводило к возрастанию температуры образца за счет ЭКЭ. После выключения поля температура образца понижалась. Величина $\Delta T$ ЭКЭ определялась как разность температур, полученных линейной экстраполяцией зависимостей $T(t)$ к моменту включения или выключения поля. Погрешность определения величины $\Delta T$ не превышала $\pm 2 \cdot 10^{-4} \mathrm{~K}$.

На рис. 8 представлены зависимости температуры от времени для трех серий экспериментов, выполненных в тетрагональной фазе в режимах включения и выключения электрического поля.

В окрестности $320 \mathrm{~K}$ надежно регистрируется обратимость процесса изменения температуры nBT (рис. 8, $a$ ). Однако в районе $340 \mathrm{~K}$ температурный ход образца после включения поля характеризуется большей скоростью $d T / d t$, чем до включения, но после выключения поля возвращается к исходной скорости (рис. 8, $b$ ). Видно, что в результате последовательности процессов $E=0 \rightarrow E \neq 0 \rightarrow E=0$ происходит необратимое увеличение температуры образца, которое растет с ростом числа циклов включения-выключения $E$. Результаты измерений при двух температурах свидетельствуют о том, 

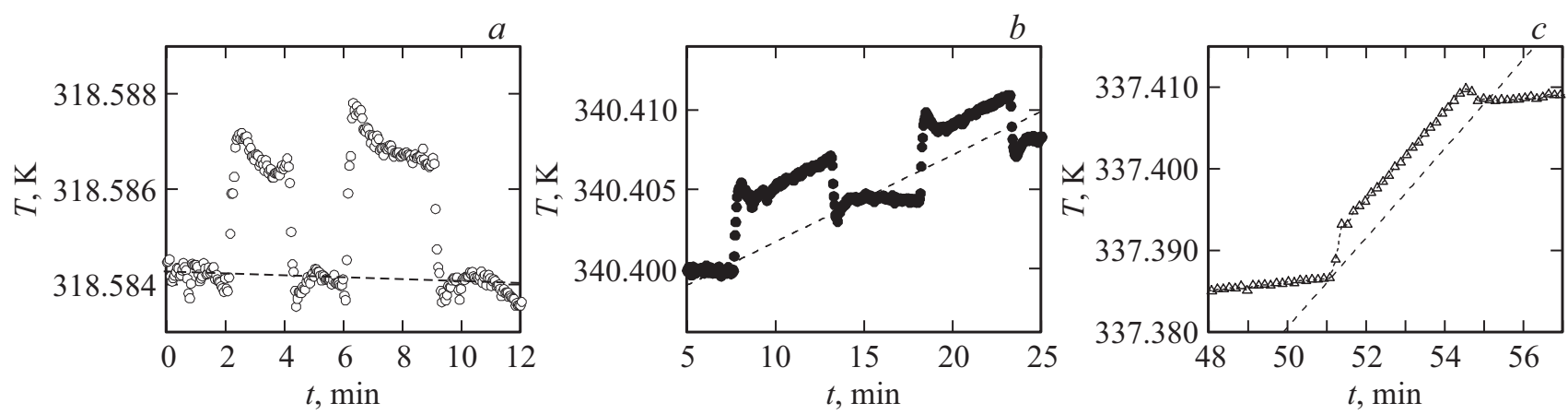

Рис. 8. Изменение температурного хода при приложении электрического поля в результате интенсивный элекрокалорический эффект при температурах около $320 \mathrm{~K}(a), 340 \mathrm{~K}(b)$ для $\mathrm{nBT}(2 \mathrm{kV} / \mathrm{cm})$ и около $340 \mathrm{~K}(c)$ для $\mathrm{mBT}(2.5 \mathrm{kV} / \mathrm{cm})$.

что увеличение скорости $d T / d t$ под полем связано с проводимостью $\mathrm{nBT}$, которая растет по мере повышения температуры. Таким образом, при $340 \mathrm{~K}$ мы наблюдали суммарное изменение температуры, связанное с ЭКЭ и выделением джоулева тепла на сопротивлении образца.

Всплески температуры при включении/выключении поля связаны с особенностями работы системы контроля и регулирования температуры. Величина этих эффектов меньше величины ЭКЭ, что позволяет надежно разделить их, путем экстраполяции температурных ходов к моментам включения-выключения $E$.

Дополнительный нагрев mBT под полем за счет джоулева тепла начинает проявляться при более низкой температуре (рис. 8,c) и, таким образом, при большем удалении от температуры ФП в кубическую фазу по сравнению с nBT. Это увеличивает трудность разделения этого эффекта и ЭКЭ, что уменьшает надежность результатов. В табл. 3 представлены данные о скоростях не связанного с ЭКЭ разогрева образцов при нескольких температурах.

Для определения действительной величины интенсивного электрокалорического эффекта $\Delta T_{\mathrm{AD}}$ необходима корректировка экспериментально измеренной величины $\Delta T_{\exp }$ поскольку теплота, выделяющаяся за счет ЭКЭ, расходуется на изменение температуры не только образца, но всей исследуемой системы образец+ячейка в целом. Связь между величинами $\Delta T_{\exp }$ и $\Delta T_{\mathrm{AD}}$ устанавливается соотношением $\Delta T_{\mathrm{AD}}=\Delta T_{\exp }\left(1+C_{f} / C_{\text {smp }}\right)$, где $C_{\text {cell }}$ - теплоемкость ячейки, определенная в отдельном эксперименте, $C_{\mathrm{smp}}$ - теплоемкость образца. Соотношение между величинами $\Delta T_{\mathrm{AD}}$ и $\Delta T_{\exp }$ для $\mathrm{nBT}$ и на $\mathrm{mBT}$ представлены в табл. 3 .

На рис. 9 показаны температурные зависимости $\Delta T_{\mathrm{AD}}$ в полях $2 \mathrm{kV} / \mathrm{cm}$ и $2.5 \mathrm{kV} / \mathrm{cm}$ соответственно для $\mathrm{nBT}$ и $\mathrm{mBT}$. Для обоих образцов надежно регистрируются максимумы ЭКЭ $\Delta T_{\mathrm{AD}}^{\max }=29 \mathrm{mK}$ при $T^{\max } \approx 410 \mathrm{~K}(\mathrm{nBT})$ и $\Delta T_{\mathrm{AD}}^{\mathrm{max}}=70 \mathrm{mK}$ при $380-390 \mathrm{~K}(\mathrm{mBT})$. В случае $\mathrm{nBT}$ температура максимума существенно превышает температуру $Ф П T_{1}=383 \mathrm{~K}$, в то время как для $\mathrm{mBT} T^{\max }$ ниже соответствующей температуры ФП $T_{1}=396.7 \mathrm{~K}$. К сожалению, более полно и надежно исследовать

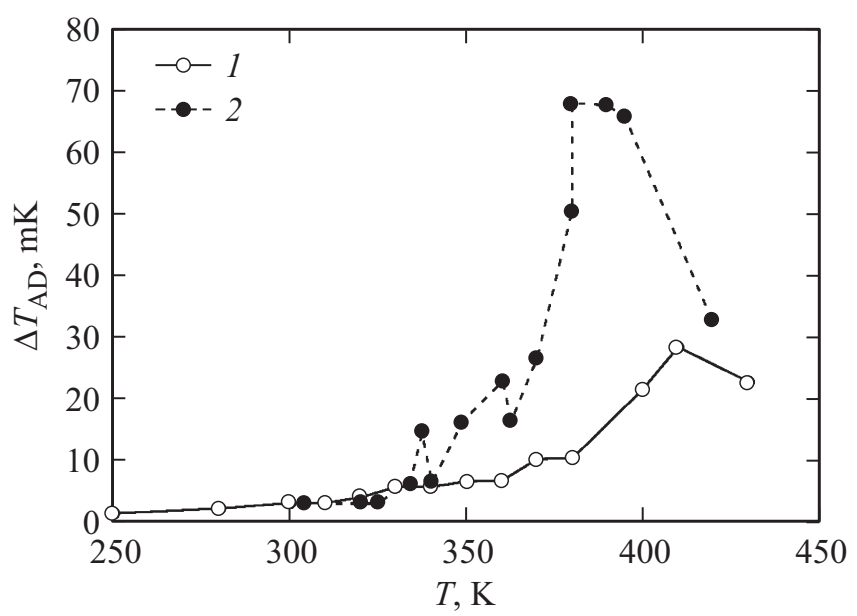

Рис. 9. Температурная зависимость интенсивного элекрокалорического эффекта $\mathrm{nBT}$ (1) и $\mathrm{mBT}(2)$.

область температур выше 400 K было невозможно из-за высокой проводимости и ограниченной предельной температуры $(T<430 \mathrm{~K})$ работы адиабатического калориметра.

\section{5. Исследование проводимости nBT}

Следует заметить, что необратимый разогрев образца за счет джоулева тепла наблюдался и при исследовании ЭКЭ в монокристаллах и слоистых керамических структурах [35,36]. Наличие относительно большой проводимости затрудняет не только определение действительной величины эффекта, но и практическое использование сегнетоэлектриков в качестве твердотельных хладагентов.

На рис. 10 представлены вольтамперные зависимости для нанокерамического образца при двух температуpax. Из явно нелинейного характера поведения следует, что электрическое сопротивление является функцией приложенного напряжения. Причина такого поведения электрического сопротивления, как правило, кроется в физических процессах на электрических контактах с диэлектриком, а также в наличии вакансий по кислороду и 


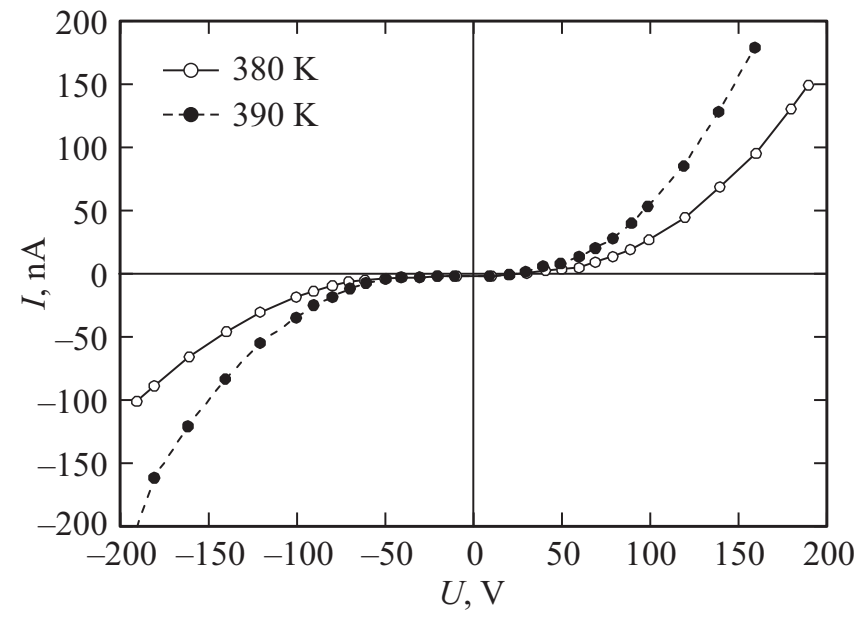

Рис. 10. Вольт-амперная характеристика $\mathrm{nBT}$ при температуpax 380 К и $390 \mathrm{~K}$.

свободных зарядов, локализованных в межзеренных границах и в самих зернах в результате спекания методом SPS [23,24,26]. Как было установлено [37], нанокерамика высокой плотности обладает проводимостью $p$-типа в зернах и высокоомными межзерновыми границами. Таким образом, в данном материале реализуется эффект на контактах между поверхностями, аналогичный процессу происходящему на параллельно-встречно включенных $p-n$ переходах в полупроводниках.

\section{4. Заключение}

Методом адиабатического калориметра выполнены исследования интенсивного ЭКЭ $\Delta T_{\mathrm{AD}}$ в окрестностях фазовых переходов в нано- и в микрокерамиках $\mathrm{BaTiO}_{3}$. Изучены некоторые аспекты влияния способов получения, термической обработки керамических образцов и размера кристаллитов на тепловые, диэлектрические и транспортные свойства, а также на величину и степень обратимости ЭКЭ.

Результаты измерения тепловых (тепловое расширение, теплоемкость) и диэлектрических свойств нано- и микрокерамик демонстрируют существенное размытие фазовых переходов в нанокерамике по сравнению с микрокерамикой, что может быть связано с уменьшением размеров зерен в нанокерамическом образце. Полное изменение энтропии в результате всех фазовых переходов в nBT уменьшилось в два раза по сравнению с mBT.

Диэлектрическая проницаемость нано и микрокерамик существенно отличается (в 30 раз) вблизи высокотемпературного фазового перехода. Такое огромное отличие в $\varepsilon(T)$ связано с методикой получения соответствующих керамик и является результатом как не стехиометрии по кислороду нанокерамического образца, так и наличием свободных зарядов на границах нанозерен, которые образуются при достаточно низкой темпе- ратуре в разреженной атмосфере в процессе спекания керамики SPS-методом.

Результаты прямых измерений ЭКЭ в адиабатических условиях в режимах включения и выключения электрического поля при низких температурах и малых электрических полях, характеризуются высокой степенью воспроизводимости и обратимости эффекта. Обнаруженная в экспериментах проводимость нано- и микрокерамик является причиной выделения джоулева тепла в образце и необратимого вклада в изменение его температуры. Наличие проводимости, на наш взгляд, может быть связано как с плохим интерфейсом между исследуемыми образцами и электродами, так и с наличием вакансий по кислороду и присутствием свободных зарядов локализованных, как в зернах, так и в межзеренных границах. К сожалению, нам не удалось реализовать ЭКЭ в сильных электрических полях в нанокерамике по причине высокой проводимости материала. Определенные величины электрокалорического коэффициента для nBT и mBT оказались значительно ниже по сравнению с монокристаллическими и поликристалическими образцами $\mathrm{BaTiO}_{3}[11,38]$.

Таким образом, результаты работы показывают, что, несмотря на различные методы получения керамик $\mathrm{BaTiO}_{3}$, наличие в них проводимости не позволяет реализовать в таких материалах значительный электрокалорический эффект в сильных электрических полях. Однако, учитывая, что все данные получены в полях низкой напряженности, безусловно, представляют интерес и являются перспективными дальнейшие исследования керамик с очень низкой проводимостью и высокой восприимчивостью к сильным электрическим полям. Разработка новых методик синтеза таких материалов будет способствовать созданию калорического элемента охлаждающих систем на основе электрокалорического эффекта.

\section{Список литературы}

[1] Y. Liu, J.F. Scott, B. Dkhil. Appl. Phys. Rev. 3, 031102 (2016).

[2] Y. Liu, J.F. Scott, B. Dkhil. Appl. Mater. 4, 064109 (2016).

[3] H. Khassaf, T. Patel, P. Alpay. J. Appl. Phys. 121, 144102 (2017).

[4] Y.V. Sinyavski, G.E. Lugansky, N.D. Pashov. Cryogenics 32, 28 (1992).

[5] S.G. Lu, B. Rožič, Q.M. Zhang, Z. Kutnjak, R. Pirc, M. Lin. Appl. Phys. Lett. 97, 2291 (2010).

[6] X. Hao, Z. Yue, J. Xu, S. An, C.-W. Nan. J. Appl. Phys. 110, 064109 (2011).

[7] D. Saranaya, A.R. Chaudhuri, J. Parui, S.B. Krupanidhi. Bull. Mater. Sci. 32, 259 (2009).

[8] A.S. Mischenko, Q.M. Zhang, J.F. Scott, R.W. Whatmore, N.D. Mathur. Science 311, 1270 (2006).

[9] S. Kar-Narayan, N.D. Mathur. J. Phys. D 43, 032002 (2010).

[10] N.A.S. Smith, M.K. Rokosz, T.M. Correia. J. Appl. Phys. 116, 044511 (2014). 
[11] X. Moya, E. Stern-Taulats, S. Crossley, D. Gonzolez-Alonso, S. Kar-Narayan, A. Planes, L. Manosa, N.D. Mathur. Adv. Mater. 25, 1360 (2013).

[12] M. Valant, A.-K. Axelsson, F. Goupil, N.M. Alford. Mater. Chem. Phys. 136, 277 (2012).

[13] D.-H. Kim, W.-S. Um, H.-G. Kim. J. Mater. Res. 11, 2002 (1996).

[14] T. Tunkasiri, G. Rujijanagul. J. Mater. Sci. Lett. 15, 1767 (1996).

[15] C. Neusel, G.A. Schneider. J. Mech. Phys. Solids 63, 201 (2014).

[16] Z. Zhao, V. Buscaglia, M. Viviani, M.T. Buscaglia, L. Mitoseriu, A. Testino, M. Nygren, M. Joknsson, P. Nanni. Phys. Rev. B 70, 024107 (2004).

[17] S. Lin, T. Lu, C. Jin, X. Wang. Phys. Rev. B 74, 134115 (2006).

[18] B.A. Strukov, S.T. Davitadze, S.N. Kravchun, S.A. Taraskin, M. Golyzman, V.V. Lemanov, S.G. Shulman. J. Phys.: Condens. Matter 15, 4331 (2003).

[19] Z. Valdez-Nava, S. Guillemet-Fritsch, C. Tenailleau, T. Lebey, B. Durand, J.Y. Chane-Ching. J. Electroceram. 22, 238 (2009).

[20] Bruker AXS TOPAS V4: General profile and structure analysis software for powder diffraction data. User's Manual (2008).

[21] G.H. Kwei, A.C. Lawson, S.J.L. Billinge, S.W. Cheong. J. Phys. Chem. 97, 2368 (1993).

[22] A.V. Kartashev, I.N. Flerov, N.V. Volkov, K.A. Sablina, Phys. Solid State 50, 2115 (2008).

[23] C. Voisin, S. Guillemet-Fritsch, P. Dufour, C. Tenailleau, H. Han, J.C. Nino. Int. J. Appl. Ceram. Technol. 10, E122 (2013).

[24] H. Han, C. Voisin, S. Guillemet-Fritsch, P. Dufour, C. Tenailleau, C. Turner, J.C. Nino. J. Appl. Phys. 113, 024102 (2013).

[25] M.A. Subramanian, D. Li, N. Duan, B.A. Reisner, A.W. Sleight. J. Solid State Chem. 151, 323 (2000).

[26] T. Takeuchi, E.B. Etourne, M. Tabuchi, H. Kageyama, Y. Kobayashi, A. Coast, F. Morrison, D.C. Sinclair, A.R. West. J. Matter. Sci. 34, 917 (1999).

[27] M. Legallais, S. Fourcade, U.-C. Chung, D. Michau, M. Maglione, F. Mauvy, C. Elissalde. J. Eur. Ceram. Soc. 38, 543 (2018).

[28] Y. He. Thermochim. Acta 419, 135 (2004).

[29] V. Mueller, L. Jager, H. Beige, H.-P. Abicht, T. Muller. Solid State Commun. 129, 757 (2004).

[30] B.A. Strukov, S.T. Davitadze, V.V. Lemanov, S.G. Shulman, Y. Uesu, S. Asanuma. Ferroelectrics 347, 179 (2007).

[31] S. Kallaev, Z. Omarov, A. Bakmaev, K. Abdulvakhidov. Phys. Solid State 55, 1095 (2013).

[32] S.F. Karmanenko, O.V. Pakhomov, A.M. Prudan, A.S. Starkov, A. Eskov. J. Eur. Ceram. Soc. 27, 3109 (2007).

[33] G. Suchaneck, G. Gerlach. Ferroelectrics 516, 1 (2017).

[34] V. Bondarev, E. Mikhaleva, I. Flerov, M. Gorev. Phys. Solid State 59, 1118 (2017).

[35] V. Bondarev, I. Flerov, M. Gorev, E. Pogoreltsev, M. Molokeev, E. Mikhaleva, A. Shabanov, A. Es'kov. Scripta Mater. 146, 51 (2018).

[36] I. Flerov, E. Mikhaleva. Phys. Solid State 50, 478 (2008)

[37] X. Guo, C. Pithan, C. Ohly, C.-L. Jia, J. Dornseiffer, F.-H. Haegel, R. Waser. Appl. Phys. Lett. 86, 082110 (2005).

[38] A.I. Karchevskii. Sov. Phys. 3, 2249 (1962).

Редактор Т.Н. Василевская 